\title{
Abnormal resting-state functional connectivity of amygdala subregions in patients with obstructive sleep apnea
}

This article was published in the following Dove Press journal:

Neuropsychiatric Disease and Treatment

\author{
Honghui Yu' \\ Liting Chen ${ }^{2}$ \\ Haijun Li ${ }^{\prime}$ \\ Huizhen Xin' \\ Juan Zhang' \\ Zhipeng Wei' \\ Dechang Peng' \\ 'Department of Radiology, the First \\ Affiliated Hospital, Nanchang University, \\ Nanchang, Jiangxi, People's Republic of \\ China; ${ }^{2}$ Department of Radiology, the \\ First Affiliated Hospital, Jinan University, \\ Guangzhou, Guangdong, People's \\ Republic of China
}

Background: The amygdala is one of the core areas of the emotional circuits. Previous neuroimaging studies have revealed that patients with obstructive sleep apnea (OSA) have aberrant structure and function in several brain areas (including the amygdala). However, the resting-state functional connectivity (rs-FC) of amgydala subregions remains uncertain.

Objective: To determine whether aberrant rs-FC exists between the amygdala subregions and other brain areas and whether such abnormalities are related to emotional disorders and cognitive impairment in OSA.

Methods: The resting-state functional magnetic resonance imaging (rs-fMRI) data of 40 male severe OSA patients and 40 matched healthy controls (HCs) were collected. The rsFC between the amygdala subregions and other brain areas was compared between the two groups. The correlations between aberrant rs-FC and clinical variables and neuropsychological assessments were evaluated.

Results: Compared with the HCs, the OSA patients showed significantly increased rs-FC between the left dorsal amygdala (DA) and the anterior lobe of the cerebellum, among the left ventrolateral amygdala (VA), the left inferior frontal gyrus (IFG) and the left superior temporal gyrus (STG), and between the right VA and the left IFG. However, significantly decreased rs-FC was observed between the right DA and the right prefrontal cortex (PFC) in OSA patients. No regional differences in rs-FC were found between the OSA patients and HCs in the bilateral medial amygdala (MA).

Conclusion: In this study, male severe OSA patients showed complex rs-FC patterns in the amygdala subregions, which may be the result of OSA-related selective damage to the amygdala, and abnormal rs-FC between the amygdala subregions and brain regions associated with emotional, cognitive and executive functions may partly explain the affective deficits and cognitive impairment observed in male severe OSA patients.

Keywords: obstructive sleep apnea, amygdala subregion, resting-state functional connectivity, functional magnetic resonance imaging, blood oxygen-level-dependent

\section{Introduction}

Obstructive sleep apnea (OSA) is a common sleep disorder characterized by recurrent breathing cessation and/or reduced airflow resulting from collapse of the upper airway, which is due to relaxation of the throat muscles and tongue and results in partial or complete obstruction of the upper respiratory tract. ${ }^{1,2}$ OSA is associated with intermittent hypoxemia, ${ }^{3}$ sleep fragmentation, ${ }^{4}$ repetitive arousals, oxygen desaturation and hypercapnia. An epidemiological investigation found that the prevalence of moderate to severe
Department of Radiology, the First Affiliated Hospital of Nanchang University, No. 17, Yongwai Zheng Street,

Donghu District, Nanchang, Jiangxi

Province 330006, People's Republic of China

Tel +8679188694457

Email pengdcdoctor@163.com 
OSA was as high as 12\% (females) and 30\% (males) among individuals 30-70 years old, and the prevalence increased as the population aged and obesity increased. ${ }^{5}$ Additionally, OSA is a multisystem chronic disease that is often associated with a variety of other diseases, such as cardiovascular disease, ${ }^{6}$ hypertension, stroke, chronic kidney disease, ${ }^{7}$ depression, ${ }^{8}$ anxiety, insomnia, and even Alzheimer's disease (AD). ${ }^{9}$ Furthermore, cognitive dysfunction has been observed in patients with OSA, including deficits in attention, memory, executive functions, emotion, visuospatial function, ${ }^{10}$ psychomotor speed, fine coordination, and language ability. ${ }^{11,12}$ The cognitive impairment observed in OSA patients is generally believed to be caused by adverse effects of long-term exposure to chronic intermittent hypoxia ${ }^{13}$ and sleep fragmentation. Nonetheless, the neural basis of cognitive dysfunction in OSA is still unclear.

Over the last few decades, neuroimaging techniques have developed into indispensable tools that are widely used in the study of various diseases, and different MRI techniques have been used to explore alterations in structure, function, and metabolism and the potential causes of cognitive impairment in OSA patients. In previous studies, OSA patients showed alterations in gray matter volume $(\mathrm{GMV})^{14,15}$ and the gray matter concentration (GMC) ${ }^{16}$ in different brain regions, and the white matter integrity of the fibers was also impaired. ${ }^{17}$ In addition, some experiments found aberrant metabolism and regional cerebral blood flow (rCBF) in OSA patients. ${ }^{18-20}$ Furthermore, aberrant spontaneous brain activity in the whole brain or regional areas has been confirmed through resting-state functional magnetic resonance imaging (rs-fMRI) methods in patients with OSA. ${ }^{21-26}$ Published studies have identified that OSA patients have impaired attention, memory, emotion, and executive functions, which are correlated with multiple brain regions including the amygdala, insular cortex, cingulate cortex, frontal regions, thalamus, temporal region, hippocampus, cerebellum, and precuneus. ${ }^{10,27}$

Joo found that a reduced GMC in the limbic areas (including the amygdala) may explain the memory and affective impairments associatedwith severe OSA. ${ }^{16}$ A metaanalysis found structural atrophy and functional disturbances in the right basolateral amygdala/hippocampus, ${ }^{28}$ and these changes were associated with the dysfunction of emotional, sensory, and limbic processes in OSA patients. Altered functional connectivity (FC) has also been shown in the insular cortex, cingulate gyrus, frontal lobe, hippocampus, and amygdala, and in areas involved in affective symptoms, such as depression and anxiety, in OSA patients. ${ }^{29}$
Studies have confirmed that the amygdala is one of the core areas involved in emotional circuits and plays an important role in emotion processing and mediating fear responses. ${ }^{30-32}$ Interestingly, for several decades, the amygdala was thought to function as a whole, which may mask the contributions of a single subdivision of the amygdala. Currently, the amygdala is generally believed to be composed of structurally and functionally heterogeneous nuclei, which have been examined in animals and humans. ${ }^{32-35}$ Cytoarchitectonic mapping of 10 human postmortem brain specimens yielded probabilistic maps of three amygdala subregions: the laterobasal (LB), centromedial (CM), and superficial (SF) subregions. ${ }^{36}$ This was the first study to demonstrate functionally distinct amygdala subdivisions in the human brain. Several neuroimaging studies have also revealed structural ${ }^{37}$ and functional ${ }^{38-40}$ distinctions in the human amygdala similar to those observed in animals. Bickart et $\mathrm{al}^{41}$ also divided the amygdala into three subregions and located the corresponding Montreal Neurological Institute (MNI) coordinates using rs-fMRI: the dorsal amygdala $( \pm 22,-4,-12)$, medial amygdala $( \pm 14,-4$, $-20)$, and ventrolateral amygdala $( \pm 28,-4,-22)$, which are defined as the DA, MA and VA in this paper, respectively.

OSA patients typically show worse depressive symptoms and higher levels of anxiety compare to individuals without $\mathrm{OSA}^{42}$ and OSA patients with depression and anxiety show greater brain damage in certain brain regions compare to patients without the same symptoms. ${ }^{43,44}$ Some studies have found that depression and anxiety symptoms in OSA patients are associated with injury in brain areas involved in affective disorders, such as the amygdala, frontal regions, hippocampus, and insular and cingulate cortices. ${ }^{17,43-45}$ Therefore, based on the above research and literature review, we believe that the amygdala has altered rs-FC in OSA patients. In our study, we used an rs-FC approach to explore the altered connectivity of the amygdala subregions with all other brain areas and to analyse the role of the amygdala in affective deficits in OSA patients. We also evaluated the relationships of aberrant rs-FC with clinical variables and neuropsychologic assessments to explore the neuroimaging mechanism of potential cognitive impairment in OSA patients.

\section{Materials and methods Subjects}

In our study, we recruited 44 newly diagnosed, untreated male patients with OSA and 42 age- and education-matched male healthy controls (HCs) from the Sleep Monitoring Room at the Respiratory Department of the First Affiliated Hospital of 
Nanchang University. Education-level matching was performed to establish a neurocognitive baseline to eliminate deviations in cognitive function between the OSA group and the $\mathrm{HC}$ group. All participants were right-handed, drug-naive, native Chinese speakers. We selected patients with severe OSA with an apnea-hypopnea index (AHI) $>30$ in this study. The inclusion criteria for OSA patients were as follows: (1) male sex, (2) AHI >30, and (3) age between 30 and 55 years. The inclusion criteria for HCs were as follows: (1) male sex, (2) AHI $<5$, and (3) age between 30 and 55 years. In addition, subjects with the following characteristics were excluded: (1) illicit drug or alcohol abuse, (2) a structural lesion on brain MRI (such as a cyst or tumor), (3) contraindications for MRI (such as claustrophobia or plate implantation), (4) a history of neurological or psychiatric diseases (such as neurodegenerative disease, epilepsy, brain trauma, depression, encephalatrophy, or brain infarction) or other sleep disorders (such as insomnia), or (5) hypertension, heart disease or diabetes. All subjects underwent clinical and neuropsychological assessments after undergoing overnight polysomnography (PSG). All participants provided written informed consent before MRI scanning and acquisition of other data, and the study protocol was approved by the Human Research Ethics Committee at The First Affiliated Hospital of Nanchang University and followed the Declaration of Helsinki.

\section{Overnight polysomnography}

All of the subjects were required to undergo overnight polysomnography (PSG) to rule out the effects of other potential sleep disorders, except for OSA. The day before PSG, the participants were required not to drink alcohol or caffeinated beverages and to avoid sedatives. Standard electroencephalogram, electrooculogram, chin electromyogram, electrocardiogram, thoracic and abdominal movements, and snoring were recorded using the Respironics LE-series physiological monitoring system (Alice 5 LE; Respironics, Orlando, FL, USA). A thermistor (to monitor nasal air flow), a nasal air pressure monitor, an oximeter (to measure oxygen saturation), piezo-electric belts (to monitor abdominal and chest movements), and a body position sensor were attached to the patients. The subjects' performances were recorded using an infrared video camera and were consecutively observed by an experienced sleep medicine physician. The subjects went to bed at 22:00 and were awakened at 06:00 next day.

In accordance with the American Academy of Sleep Medicine guidelines (AASM), obstructive apnea was defined as a continuous absence of airflow or a reduction in airflow $\geq 90 \%$ for at least $\geq 10$ s associated with evident respiratory effort. Hypopnea was defined as a reduction in airflow $\geq 30 \%$ with a $4 \%$ or higher oxygen desaturation or with electroencephalographic (EEG) arousal. The AHI was obtained from the mean durations of apnea and hypopnea per hour during sleep. OSA was diagnosed when the AHI was $\geq 5$, and severe OSA was diagnosed when the AHI was $>30$.

\section{Clinical and neuropsychological measures}

All participants with OSA and the HCs were asked to complete a self-reported sleep questionnaire using the Montreal Cognitive Assessment (MoCA, Chinese version), and the Epworth Sleepiness Scale (ESS) was administered by an experienced psychologist. The MoCA is a rapid screening evaluation tool that can assess various cognitive domains, including executive function, attention, naming, visuoconstructional skills, memory, calculation, language, abstraction and orientation. The maximum score for the MoCA is 30 , and a total score less than or equal to 26 implies the existence of a mild recognitive insult. Interestingly, one point is added for participants with less than 12 years of education compared with those who are well-educated to correct for educational deviations. The ESS, a sleep questionnaire, was used to evaluate daytime somnolence in subjects according to different categories. ESS scores range from 0 to 24 (eight different categories from 0 to 3). A score higher than 6 was defined as sleepiness, a score higher than 11 was defined as excessive sleepiness, and a score higher than 16 was defined as risky sleep.

\section{MRI data acquisition}

All participants collected both of functional and structural MRI images in a 3.0 T MRI scanner (Siemens, Erlangen, Germany) equipped with an 8-channel phased-array head coil in our hospital. Scan ranges from the skull base to the top of the head. All participants were asked to abstain from drinking wine, coffee, and sedatives the day before MRI scanning. During the scanning, subjects were lying on the scanning bed with their eyes closed, and required to stay awake, to be quiet, to be relax, and to think of nothing in particular. Foam pads were used to minimize head motion of factitious factors and ear plugs were used to minimize noise of MRI scanning machine. Rs-fMRI data were acquired with a gradient-recalled echo planar imaging (EPI) sequence in the axial plane: repetition time $(\mathrm{TR})=2,000 \mathrm{~ms}$, echo time $(\mathrm{TE})=30 \mathrm{~ms}$, flip angle $=90^{\circ}$, 
thickness $=4.0 \mathrm{~mm}$, gap $=1.2 \mathrm{~mm}$, feld-of-view (FOV) $=230 \times 230 \mathrm{~mm}^{2}$, matrix size $=64 \times 64$, slices $=30 ; 240$ rsfMRI images totally were recorded. High-resolution T1weighted brain structural MRI images were obtained from each subject using a magnetization-prepared rapid gradient echo pulse sequence in the sagittal plane: $\mathrm{TR}=1,900 \mathrm{~ms}$, $\mathrm{TE}=2.26 \mathrm{~ms}$, flip angle $=90^{\circ}$, thickness $=1.0 \mathrm{~mm}$, gap $=0.5 \mathrm{~mm}, \mathrm{FOV}=250 \times 250 \mathrm{~mm}^{2}$, resolution matrix size $=256 \times 256$, slices $=176$. Otherwise, regular T1weighted $(\mathrm{TR}=250 \mathrm{~ms}, \mathrm{TE}=2.46 \mathrm{~ms}$, thickness $=5 \mathrm{~mm}$, gap $=1.5 \mathrm{~mm}, \mathrm{FOV}=220 \times 220 \mathrm{~mm}^{2}$, slices=19) and T2weighted $(\mathrm{TR}=4,000 \mathrm{~ms}, \mathrm{TE}=113 \mathrm{~ms}$, thickness $=5 \mathrm{~mm}$, gap $=1.5 \mathrm{~mm}, \mathrm{FOV}=220 \times 220 \mathrm{~mm}^{2}$, slices=19) images were collected to exclude obvious brain lesions performed by two senior radiologists. No participants were excluded because of brain lesions.

\section{Data preprocessing}

fMRI data preprocessing was carried out using the Data Processing \& Analysis Assistant for RestingState Brain Imaging (DPABI, Chinese Academy of Sciences, Beijing, China, http://www.restfmri.net) ${ }^{46}$ based on the MATLAB2012a (Math Works, Natick, MA, USA) and statistical parametric mapping package (SPM8, Wellcome Department of Cognitive Neurology, London, UK) platform.

1. File format converted from DICOM to NIFTI;

2. Removal of the first ten time points of each participant due to the instability of the premier MRI signal and the participants adapting to the noise of MRI scanning machine;

3. Slice timing correction was performed in the remaining 230 volumes;

4. Head motion parameters were computed by estimating translation $(\mathrm{mm})$ in each direction and the angular rotation (degree) on each axis for each voxel. The head motion criteria was maximum translation less than $2.0 \mathrm{~mm}$ and maximum rotation less than $2.0^{\circ}$ in our study, all the subjects well-cooperated and no one was rejected by the standard. Additionally, subjects were excluded if they had a frame-wise displacement (FD) with more than 2.5 standard deviations, and four patients with OSA and two HCs were excluded according to the standard of head motion;

5. To ensure a correct normalization process, we used the Diffeomorphic Anatomical Registration Through Exponentiated Lie Algebra (DARTEL) ${ }^{47}$ toolbox to create customized templates by $\mathrm{T} 1$ structural images of all subjects, and consequently used the optimized templates to segment all subjects' structural images into white matter, and cerebrospinal fluid (CSF);

6. Functional data were spatially normalized to the MNI template, and all images were then subsampled to $3 \times 3 \times 3 \mathrm{~mm}^{3}$ voxels and the resulting data was spatially smoothed with a $6 \mathrm{~mm}$ fullwidth at half-maximum (FWHM) Gaussian kernel;

7. Temporal band-pass filtering (0.01-0.08 Hz) was performed to minimize the effect of low-frequency drift and high-frequency noise;

8. A multiple regression method was performed to further reduce possible sources of artifacts, including the nuisance signal (white matter, CSF, and global signal) and the Friston 24-parameter, ${ }^{48}$ and linear drift were regressed from the time series of all voxels.

\section{Functional connectivity analysis}

First, we defined six subregions of the bilateral amygdala as seed regions of interest (ROIs) according to Bickart, ${ }^{41}$ and each subregion had aradius of $3 \mathrm{~mm}$ : the DA (MNI coordinates $\pm 22,-4,-12)$, MA $( \pm 14$, $-4,-20)$, and VA $( \pm 28,-4,-22)$. Next, the average time series of the ROIs were extracted from each participant, and Pearson's correlation coefficient was computed between each ROI to obtain the rs-FC map for each subject. Finally, the Fisher r-to-z transformation was executed in the resultant correlation maps to generate a more normal distribution.

\section{Statistical analysis}

Demographic, biophysical and sleep data and neuropsychological scores were compared between the two groups using the two-samples $t$-test with Statistical Package for the Social Sciences version 24.0 (SPSS, Chicago, IL, USA). Then, we used two independentsample $t$-tests to analyze differences in the rs-FC maps among the groups by using the body mass index (BMI), age and education as covariables. Multilevel comparisons were corrected using Gaussian random field theory (GRF, two-tailed, voxel-level $P<0.01$ and cluster-level $p<0.05)$. Finally, the relationships between the average $\mathrm{z}$-values of each brain region with significant intergroup 
differences and clinical indices in OSA patients were evaluated using Pearson correlation analysis. $P<0.05$ was considered statistically significant.

\section{Results}

\section{Demographic and clinical data}

The demographic data and neuropsychological scores of the subjects are shown in Table 1 . We found no significant differences in age, total sleep time (TST), stage 2 sleep, stage $3+4$ sleep, education, and naming and orientation functions between the OSA patients and $\mathrm{HCs}(P>0.05)$. However, significant intergroup differences were observed in the BMI, AHI, nadir $\mathrm{SaO} 2$, average $\mathrm{SaO} 2$, sleep efficiency, stage 1 sleep, rapid eye movement (REM), $\mathrm{SaO} 2<90 \%$, arousal index, oxygen desaturation index (ODI), ESS score, MoCA score, and visuospatial/executive, delayed recall, attention, language, and abstraction functions.

\section{Intergroup differences in the rs-FC of the amygdala subregions}

Compared with the $\mathrm{HCs}$, we found that the rs-FC of the amygdala subregions showed significant differences in some areas in OSA patients. We found that OSA patients showed significantly increased rs-FC between the left DA and anterior lobe of the cerebellum (including vermis 4/5), among the left VA, the left inferior frontal gyrus (IFG), and the left superior temporal gyrus (STG), and between the right VA and the left IFG, while significantly decreased rs$\mathrm{FC}$ was noted between the right DA and the right prefrontal cortex (PFC). However, no regional differences in rs-FC were found between the OSA patients and HCs in the bilateral MA. Detailed information is shown in Figure 1 and Table 2.

\section{Correlation analysis}

In OSA patients, the rs-FC between the left DA and anterior lobe of the cerebellum (including the vermis 4/5) showed a positive correlation with the ODI $(r=0.359, P=0.023)$. Otherwise, the rs-FC between the left VA and the left IFG showed a negative correlation with visuospatial/executive scores and the maximum pause time $(r=-0.389, P=0.013$; $r=-0.322, P=0.043$, respectively) (Figure 2).

\section{Discussion}

In the 1930s and 1940s, several studies suggested that a system of brain structures was responsible for emotion and emotional expression, which can be summarized as the concept of the "limbic system" proposed by MacLean and Papez. ${ }^{49,50}$ They considered the "limbic system" to include the hippocampal formation (containing the amygdala), cingulate gyrus, and anterior thalamus, and the limbic system has been further defined in subsequent studies. This definition was very influential at that time, and neuroscientists once thought that the problem of how the brain generates emotions was solved by the limbic system concept. In recent decades, neuroscientists have realized that significant work was still required after decades of neglect of the study of emotions. The limbic system plays different roles in emotion, memory, behavior, cognition, learning, attention and social processing, and its most famous role is in emotion. ${ }^{51}$ As an indispensable part of the emotional circuit, the function of the amygdala was further studied.

First, we found that both the bilateral DA and VA in OSA patients had abnormal rs-FC with other areas compared to $\mathrm{HCs}$; however, the rs-FC of the bilateral MA showed no significant abnormal connectivity to any area. Bickart et $\mathrm{al}^{41}$ found that the dorsal, medial and ventrolateral amygdala were linked to networks supporting social aversion, social affiliation and social perception, which are implicated in motivating avoidant behaviors, motivating prosocial behaviors, and decoding social signals in the context of past experiences and current goals, respectively. Studies have found that the laterobasal subregion of the amygdala promotes the associative learning process of fear conditioning, ${ }^{31,32}$ the centromedial nuclei play an important role in behavioral responses, ${ }^{32,52}$ and the superficial subdivision of the amygdala is involved in olfactory and affective processes. $^{53-55}$ Because the amygdala has a complex structure and functions, the amygdala regions tend to be functionally specialized; thus, we speculated that the bilateral MA would not have aberrant rs-FC with any brain area, possibly because OSA causes selective damage to different amygdala subregions.

Previous anatomical studies have found structural connections between the amygdala and multiple brain regions. ${ }^{56}$ For example, the amygdala and the PFC have extensive structural connections in addition to functional connections, especially the medial PFC (mPFC). ${ }^{57,58}$ On the other hand, several studies have demonstrated that emotional regulation is caused by top-down inhibition of the PFC on the amygdala. ${ }^{59,60}$ The FC between the PFC and the amygdala is called the amygdala-frontal loop, ${ }^{59,61}$ which plays an important role in the production and regulation of emotion. Furthermore, sleep-deprived patients 
Table I Demographic and clinical characteristics of OSA and HCs

\begin{tabular}{|c|c|c|c|c|}
\hline Characteristic & $\operatorname{OSA}(n=40)$ & $\operatorname{HCs}(n=40)$ & t-value & $P$-value \\
\hline Age, year & $37.03 \pm 8.74$ & $38.58 \pm 12.16$ & -0.655 & 0.515 \\
\hline BMI & $27.40 \pm 3.47$ & $23.08 \pm 1.96$ & 6.859 & $<0.00 I^{*}$ \\
\hline $\mathrm{AHI}$ & $60.15 \pm 20.45$ & $2.5 I \pm I .2 I$ & 17.795 & $<0.00 I^{*}$ \\
\hline Nadir SaO2, \% & $66.00 \pm 12.68$ & $90.25 \pm 3.05$ & -11.762 & $<0.00 I^{*}$ \\
\hline Average $\mathrm{SaO} 2, \%$ & $90.83 \pm 4.49$ & $95.58 \pm 2.56$ & -5.813 & $<0.00 I^{*}$ \\
\hline TST, min & $373.80 \pm 76.33$ & $398.28 \pm 19.98$ & -1.962 & 0.053 \\
\hline Sleep efficiency, \% & $84.58 \pm 16.54$ & $92.18 \pm 5.40$ & -2.763 & $0.007^{*}$ \\
\hline Stage I, \% & $29.68 \pm 15.65$ & $10.43 \pm 3.78$ & 7.563 & $<0.00 I^{*}$ \\
\hline Stage $2, \%$ & $39.81 \pm 13.55$ & $39.75 \pm 6.11$ & 0.024 & $0.98 I$ \\
\hline Stage $3+4, \%$ & $22.83 \pm 17.74$ & $21.42 \pm 4.56$ & 0.483 & 0.630 \\
\hline REM, \% & $7.53 \pm 8.36$ & $22.13 \pm 7.91$ & -8.024 & $<0.00 I^{*}$ \\
\hline $\mathrm{SaO} 2<90 \%$ & $32.68 \pm 21.00$ & $0.28 \pm 0.17$ & 9.759 & $<0.00 I^{*}$ \\
\hline Arousal index & $41.33 \pm 24.73$ & $11.95 \pm 2.84$ & 7.465 & $<0.00 I^{*}$ \\
\hline ODI & $55.65 \pm 25.84$ & $2.74 \pm 1.42$ & 12.930 & $<0.00 I^{*}$ \\
\hline Education, years & $12.40 \pm 2.74$ & $12.18 \pm 2.73$ & 0.368 & 0.714 \\
\hline ESS score & $11.98 \pm 3.77$ & $3.45 \pm 2.05$ & 12.576 & $<0.00 I^{*}$ \\
\hline Global MoCA score & $25.08 \pm 2.17$ & $27.73 \pm 1.40$ & -6.057 & $<0.00 I^{*}$ \\
\hline MoCA: Visuospatial/ & $4.03 \pm 0.83$ & $4.65 \pm 0.66$ & -3.718 & $<0.00 I^{*}$ \\
\hline \multicolumn{5}{|l|}{ Executive } \\
\hline MoCA: Naming & $2.95 \pm 0.22$ & $3.00 \pm 0$ & -1.433 & 0.156 \\
\hline MoCA: Delay recall & $3.15 \pm 1.19$ & $4.83 \pm 0.39$ & -8.479 & $<0.00 I^{*}$ \\
\hline MoCA: Attention & $5.28 \pm 1.04$ & $5.83 \pm 0.39$ & -3.144 & $0.002 *$ \\
\hline MoCA: Language & $2.03 \pm 0.53$ & $2.80 \pm 0.4 I$ & -7.343 & $<0.00 I^{*}$ \\
\hline MoCA: Abstraction & $1.48 \pm 0.5 \mathrm{I}$ & $1.83 \pm 0.39$ & -3.483 & $0.001 *$ \\
\hline MoCA: Orientation & $5.70 \pm 0.69$ & $5.93 \pm 0.27$ & -1.931 & 0.057 \\
\hline
\end{tabular}

Note: ${ }^{*} P<0.05$, which was considered statistically signifcant.

Abbreviations: OSA, obstructive sleep apnea; HCs, healthy controls; BMI, body mass index; AHI, apnea hypopnea index; TST, total sleep time; REM, rapid eye movement; ODI, oxygen desaturation index; $\mathrm{SaO} 2<90 \%$, percentage of total sleep time spent at an oxygen saturation less than $90 \%$; ESS, epworth sleepiness scale; MoCA, montreal cognitive assessment; $\mathrm{n}$, numbers.

have decreased FC between the amygdala and mPFC, which suggesting that sleep deprivation may lead to an enhanced response in the amygdala to negative emotional stimuli and a reduction in top-down control. ${ }^{62}$ Liu found that patients with OSA had decreased FC between the posterior cingulate cortex (PCC) and the $\mathrm{MPFC}$; thus, the decreased FC with the MPFC may be related to an affective deficit. ${ }^{63}$ Consistent with these findings, our study revealed that OSA patients showed decreased rs-FC between the right $\mathrm{DA}$ and the right $\mathrm{PFC}$, suggesting that the inhibitory effect of the PFC on the amygdala was weakened, which may have led to an increase in negative emotions and may be the potential cause of emotional dysfunction in OSA patients. Furthermore, we also found that OSA patients showed increased rs-FC between the bilateral VA and the left IFG, reflecting a potential adaptive compensatory mechanism for the decline in emotionrelated function and mobilization of additional cognitive resources to suppress the influence of negative emotion in
OSA patients. In addition, the frontal lobe is an important brain region involved in multiple cognitive functions. Abnormal rs-FC between the amygdala and the frontal lobe may be related to cognitive impairment in OSA patients, such as impairments in working memory, attention, learning and executive functions, ${ }^{10}$ which may partly explain why the rs-FC between the left VA and left IFG showed a significant negative correlation with visuospatial/executive scores in this study.

The temporal lobe is closely connected to the limbic cortex and is mainly responsible for the processing of auditory information, language understanding, and memory and emotion. In particular, the STG plays an important role in emotional processing and social cognition. ${ }^{64,65}$ Furthermore, Lim found that temporal activities are processed by the orbitofrontal-temporal lobe-limbic system. ${ }^{66}$ In a previous study, reductions in GMV and GMC in the temporal lobe ${ }^{14}$ and increased rs-FC between the left temporal gyrus and the PCC were found in OSA patients, 


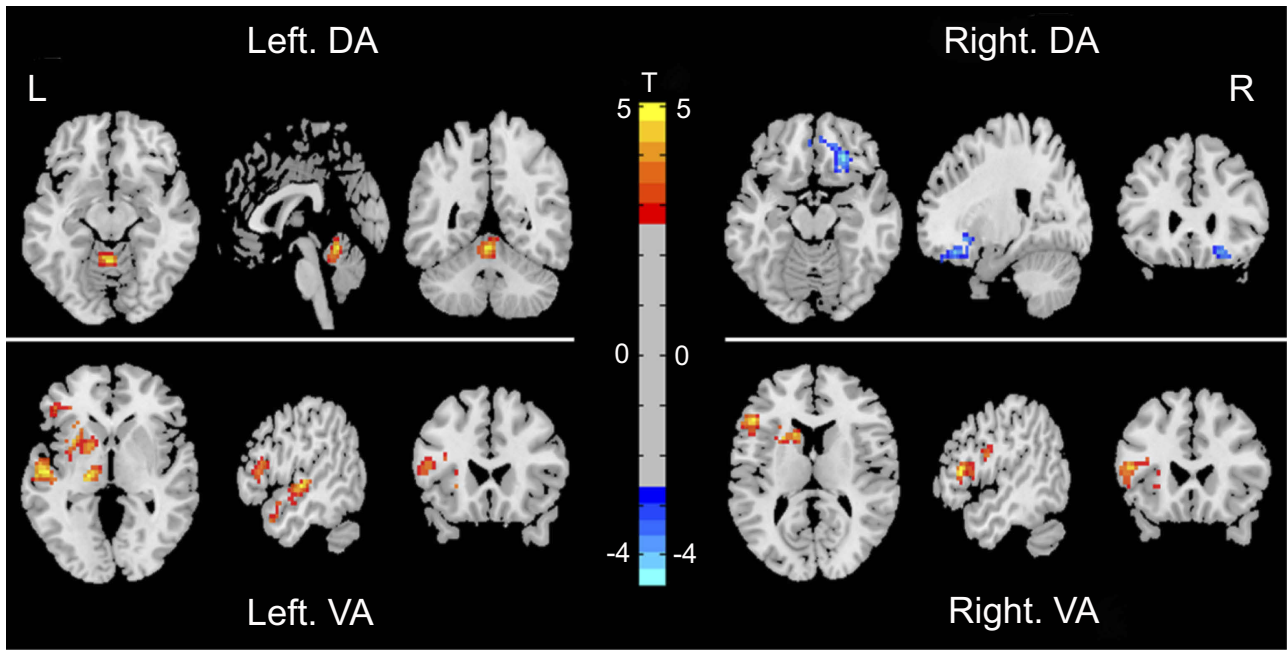

Figure I The differences map in rs-FC between OSA patients and HCs (two-tailed GRF correction, voxel-level $P<0.0 \mathrm{I}$, and cluster-level $P<0.05$ ). The blue region represents decreased rs-FC, and red region represents increased $\mathrm{rs}-\mathrm{FC}$.

Table 2 Intergroup differences in the rs-FC of the amygdala subregions

\begin{tabular}{|c|c|c|c|c|c|}
\hline \multirow[t]{2}{*}{ Brain regions } & \multicolumn{3}{|c|}{$\begin{array}{l}\text { MNI } \\
\text { coordinates }\end{array}$} & \multirow[t]{2}{*}{$\begin{array}{l}\text { Cluster } \\
\text { size }\end{array}$} & \multirow[t]{2}{*}{ T-value } \\
\hline & $\mathbf{x}$ & $y$ & $\mathbf{z}$ & & \\
\hline $\begin{array}{l}\text { Seed:Left DA } \\
\text { cerebellum anterior } \\
\text { lobe (including vermis } \\
4 / 5 \text { ) }\end{array}$ & 0 & -51 & -12 & 111 & 4.21 \\
\hline $\begin{array}{l}\text { Seed:Right DA } \\
\text { right PFC }\end{array}$ & 24 & 33 & -15 & 111 & -3.99 \\
\hline $\begin{array}{l}\text { Seed:Left VA } \\
\text { left IFG } \\
\text { left STG }\end{array}$ & $\begin{array}{l}-51 \\
-54\end{array}$ & $\begin{array}{l}24 \\
-15\end{array}$ & $\begin{array}{l}12 \\
0\end{array}$ & $\begin{array}{l}109 \\
420\end{array}$ & $\begin{array}{l}3.67 \\
4.88\end{array}$ \\
\hline $\begin{array}{l}\text { Seed:Right VA } \\
\text { left IFG }\end{array}$ & -48 & 27 & 12 & 314 & 5.10 \\
\hline
\end{tabular}

Abbreviations: rs- $\mathrm{FC}$, resting-state functional connectivity; $\mathrm{MNI}$, montreal neurological institute; DA, dorsal amygdala; VA, ventrolateral amygdala; PFC, prefrontal cortex; IFG, inferior frontal gyrus; STG, superior temporal gyrus.

indicating an adaptive compensatory response to cognitive impairment. ${ }^{63}$ Additionally, Nie et al $^{67}$ revealed reduced rCBF in the parahippocampus and temporal lobe in patients with OSA, which may partly explain the impairment in memory and emotional processing in these patients. Furthermore, Chen et $\mathrm{al}^{68}$ found significantly decreased rs-FC in default mode network (DMN) brain regions involving the prefrontal, parietal and temporal lobes, which may be associated with abnormal emotional symptoms in patients with OSA, such as depression and anxiety. Thus, we speculated that the abnormal rs-FC between the left VA and the left STG that we found may provide a new perspective to understand the causes of memory and affective disorders in OSA patients, and the increased rs-FC may reflect a compensatory mechanism to offset the deficits in memory and affection.

Using the rs-FC method, a previous study evaluated the rs-FC between the amygdala subregions and anterior regions of the cerebellum in healthy people with no history of psychiatric or neurological illness. ${ }^{35}$ Basic research supports anatomical connectivity between the amygdala and cerebellar vermis, ${ }^{69,70}$ and the vermis has been implicated in fear responses. ${ }^{70-72}$ Generalized anxiety disorder (GAD) patients have disruptions in the FC between the amygdala and cerebellum, which may reflect a disruption in fear learning among adolescents with GAD. ${ }^{73}$ Strong differential connectivity was also observed between the CM subregion of the amygdala and the cerebellum (especially the vermis) in patients with GAD, which supports the evidence that the cerebellum, in particular the vermis, plays a role in emotional processes through connectivity with the CM subregion of the amygdala. ${ }^{74}$ Therefore, the increased rs-FC between the left DA and the anterior lobe of the cerebellum (including the vermis 4/5) may be associated with emotional dysfunction in OSA patients, such as fear conditions; however, this findings remains to be confirmed by further research. We found that OSA patients showed a markedly higher ODI than HCs that was positively correlated with the rs-FC between the left DA and the anterior lobe of the cerebellum (including the vermis4/5), 

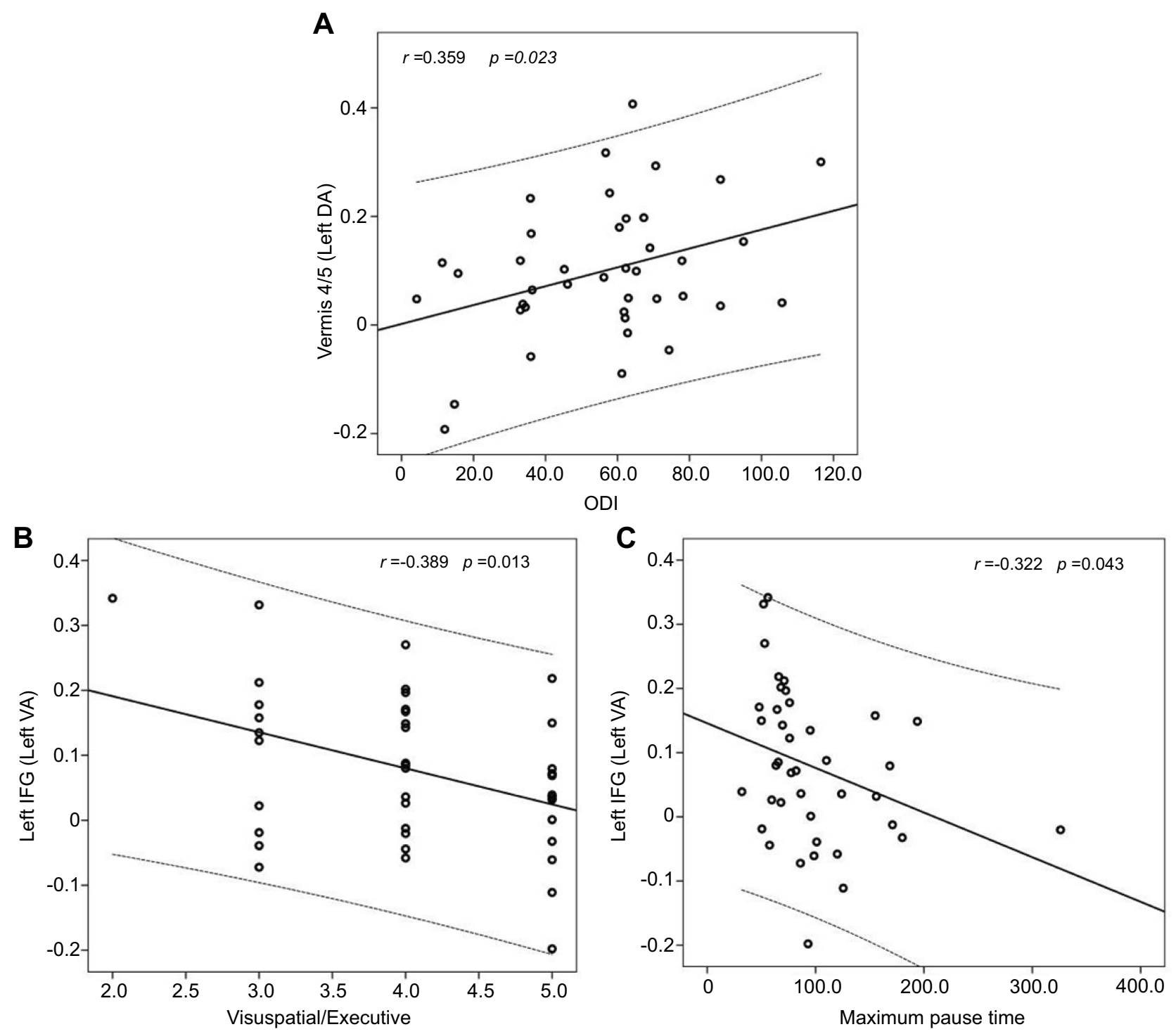

Figure 2 In OSA patients, the rs-FC between the left DA and anterior lobe of the cerebellum (including vermis 4/5) showed a positive correlation with the ODI (Fig. A), and the rsFC between the left VA and the left IFG showed a negative correlation with visuospatial/executive scores and maximum pause time (Fig. B and C).

Abbreviations: OSA, obstructive sleep apnea; rs-FC, resting-state functional connectivity; ODI, oxygen desaturation index; DA, dorsal amygdala; VA, ventrolateral amygdala; IFG, inferior frontal gyrus.

suggesting that hypoxemia, the most important damaging factor, may be the reason for the abnormal rs-FC of the amygdala in OSA patients. The cerebellum is involved in respiratory regulation, and the enhanced rs-FC between the left DA and the anterior lobe of the cerebellum (including the vermis 4/5) may be an adaptive compensatory mechanism for hypoxemia in OSA.

Unfortunately, we found that some brain regions with abnormal rs-FC were associated with some clinical or cognitive-related indicators, including the ODI $(\mathrm{r}=0.359, p=0.023)$, visuospatial/executive scores $(\mathrm{r}=-0.389, p=0.013)$ and the maximum pause time $(\mathrm{r}=-0.322, p=0.043)$. However, all of these results have small correlation coefficients, thus rendering them unreliable and difficult to explain, but we speculate that some uncontrollable factors in the data acquisition process may have led to these results. Nevertheless, the results also provided some reference value for understanding the occurrence and development of OSA.

Several limitations existed in our study. First, we recruited only male patients with severe OSA; female patients and those with mild-to-moderate OSA should be included in future studies. Second, cognitive function was assessed using only the MoCA scale, which does not include an emotional assessment. Our team initially did not intend to conduct this research, but as the study continued, we found that affective disorders in patients with 
OSA must be studied. Finally, we selected only the amygdala, one of the most important brain regions involved in the emotional loop, as the seed ROI in this study, even though this region does not represent the full picture of emotional disorders in OSA patients. Therefore, emotional assessments and other important structures of the emotional circuits should be included in future studies.

\section{Conclusion}

In this study, using a seed-based rs-fMRI method, we found aberrant rs-FC between amygdala subregions and several other brain regions, including the anterior lobe of the cerebellum (including the vermis 4/5), right PFC, left IFG and left STG, which may be the potential cause of affective deficits and cognitive impairment in male severe OSA patients.

\section{Acknowledgments}

This work was supported by grants from the Natural Science Foundation of China (Grant No. 81560285, 81860307), the Natural Science Foundation of Jiangxi province, China (Grant No. 20171BAB205070, 20181ACB20023), the Education Department Foundation of Jiangxi province, China (Grant No. 700544006), the Science and Technology Support Program of Jiangxi province, China (20141BBG70026), the Graduate Innovation Foundation of Jiangxi province, China (Grant No. YC2017-S089).

\section{Disclosure}

The authors report no conflicts of interest in this work.

\section{References}

1. Park JG, Ramar K, Olson EJ. Updates on definition, consequences, and management of obstructive sleep apnea. Mayo Clin Proc. 2011;86:549-555. doi:10.4065/mcp.2010.0810

2. Tan J, Huang J, Yang J, et al. Numerical simulation for the upper airway flow characteristics of Chinese patients with OSAHS using CFD models. Eur Arch Otorhinolaryngol. 2013;270(3):1035-1043. doi:10.1007/s00405-013-2363-x

3. Lim DC, Pack AI. Obstructive sleep apnea and cognitive impairment: addressing the blood-brain barrier. Sleep Med Rev. 2014;18(1):35-48. doi:10.1016/j.smrv.2012.12.003

4. Ferri R, Drago V, Aricò D, et al. The effects of experimental sleep fragmentation on cognitive processing. Sleep Med. 2010;11 (4):378-385. doi:10.1016/j.sleep.2010.01.006

5. Peppard PE, Young T, Barnet JH, Palta M, Hagen EW, Hla KM. Increased prevalence of sleep-disordered breathing in adults. $\mathrm{Am}$ J Epidemiol. 2013;177(9):1006-1014. doi:10.1093/aje/kws342

6. Testelmans D, Tamisier R, Barone-Rochette G, et al. Profile of circulating cytokines: impact of OSA, obesity and acute cardiovascular events. Cytokine. 2013;62(2):210-216. doi:10.1016/j.cyto.2013.02.021

7. Yayan J, Rasche K, Vlachou A. Obstructive sleep apnea and chronic kidney disease. Adv Exp Med Biol. 2017;1022:11-18.
8. Kerner NA, Roose SP. Obstructive sleep apnea is linked to depression and cognitive impairment: evidence and potential mechanisms. Am J Geriatr Psychiatry. 2016;24(6):496-508. doi:10.1016/j.jagp.2016.01.134

9. Daulatzai AM. Evidence of neurodegeneration in obstructive sleep apnea: relationship between obstructive sleep apnea and cognitive dysfunction in the elderly. J Neurosci Res. 2015;93(12):1778-1794. doi:10.1002/jnr.23634

10. Bruin PF, Bagnato MC. Cognitive impairment in obstructive sleep apnea syndrome. J Bras Pneumol. 2010;36(8):32-37.

11. Bucks RS, Olaithe M, Eastwood P. Neurocognitive function in obstructive sleep apnoea: a meta-review. Respirology. 2013;18 (1):61-70. doi:10.1111/j.1440-1843.2012.02255.x

12. Olaithe M, Bucks RS, Hillman DR, Eastwood PR. Cognitive deficits in obstructive sleep apnea: insights from a meta-review and comparison with deficits observed in COPD, insomnia, and sleep deprivation. Sleep Med Rev. 2018;38:39-49. doi:10.1016/j.smrv.2017.03.005

13. Gozal D. CrossTalk proposal: the intermittent hypoxia attending severe obstructive sleep apnea does lead to alterations in brain structure and function. J Physiol. 2013;591(2):379-381. doi:10.1113/ jphysiol.2012.247577

14. Macey PM, Henderson LA, Macey KE, et al. Brain morphology associated with obstructive sleep apnea. Am J Respir Crit Care Med. 2002;166(10):1382-1387. doi:10.1164/rccm.200201-050OC

15. Yaouhi K, Bertran F, Clochon P. A combined neuropsychological and brain imaging study of obstructive sleep apnea. J Sleep Res. 2009;18 (1):36-48. doi:10.1111/j.1365-2869.2008.00705.x

16. Joo EY, Tae WS, Lee MJ, et al. Reduced brain gray matter concentration in patients with obstructive sleep apnea syndrome. Sleep. 2010;33(2):235-241.

17. Macey PM, Kumar R, Woo MA, Valladares EM, Yan-Go FL, Harper RM. Brain structural changes in obstructive sleep apnea. Sleep. 2008;31(7):967-977.

18. Sarchielli P, Presciutti O, Alberti A, et al. A ${ }^{1} \mathrm{H}$ magnetic resonance spectroscopy study in patients with obstructive sleep apnea. Eur J Neurol. 2008;15(10):1058-1064. doi:10.1111/ j.1468-1331.2008.02244.x

19. Joo EY, Tae WS, Han SJ, Cho J-W, Hong SB. Reduced cerebral blood flow during wakefulness in obstructive sleep apnea-hypopnea syndrome. Sleep. 2007;30(11):1515-1520.

20. Kumar R, Pham TT, Macey PM, Woo MA, Yan-Go FL, Harper RM. Abnormal myelin and axonal integrity in recently diagnosed patients with obstructive sleep apnea. Sleep. 2014;37(4):723-732. doi: $10.5665 /$ sleep. 3578

21. Zhang Q, Wang D, Qin W, et al. Altered resting-state brain activity in obstructive sleep apnea. Sleep. 2013;36(5):651-659. doi:10.5665/ sleep. 2620

22. Zhang Q, Qin W, He X, et al. Functional disconnection of the right anterior insula in obstructive sleep apnea. Sleep Med. 2015;16 (9):1062-1070. doi:10.1016/j.sleep.2015.04.018

23. Zhang X, Ma L, Li S, Wang Y, Wang L. A functional MRI evaluation of frontal dysfunction in patients with severe obstructive sleep apnea. Sleep Med. 2011;12(4):335-340. doi:10.1016/j. sleep.2010.08.015

24. Li HJ, Nie X, Gong $\mathrm{HH}$, et al. Abnormal resting-state functional connectivity within the default mode network subregions in male patients with obstructive sleep apnea. Neuropsychiatr Dis Treat. 2014;12:203-212.

25. Li HJ, Dai XJ, Gong HH, et al. Aberrant spontaneous low-frequency brain activity in male patients with severe obstructive sleep apnea revealed by resting-state functional MRI. Neuropsychiatr Dis Treat. 2015;11:207-214.

26. Peng DC, Dai XJ, Gong HH, et al. Altered intrinsic regional brain activity in male patients with severe obstructive sleep apnea: a resting-state functional magnetic resonance imaging study. Neuropsychiatr Dis Treat. 2014;10:1819-1826. 
27. Torelli F, Moscufo N, Garreffa G, et al. Cognitive profile and brain morphological changes in obstructive sleep apnea. NeuroImage. 2011;54(2):787-793. doi:10.1016/j.neuroimage.2010.09.065

28. Tahmasian M, Rosenzweig I, Eickhoff SB, et al. Structural and functional neural adaptations in obstructive sleep apnea: an activation likelihood estimation meta-analysis. Neurosci Biobehav Rev. 2016;65:142-156. doi:10.1016/j.neubiorev.2016.03.026

29. Park B, Palomares JA, Woo MA, et al. Disrupted functional brain network organization in patients with obstructive sleep apnea. Brain Behav. 2016;6(3):e00441. doi:10.1002/brb3.441

30. Muller VI, Habel U, Derntl B, et al. Incongruence effects in crossmodal emotional integration. Neuroimage. 2011;54(3):2257-2266. doi:10.1016/j.neuroimage.2010.10.047

31. Phelps EA, Ledoux JE. Contributions of the amygdala to emotion processing: from animal models to human behavior. Neuron. 2005;48 (2):175-187. doi:10.1016/j.neuron.2005.09.025

32. Ledoux J. The emotional brain, fear, and the amygdala. Cell Mol Neurobiol. 2003;23(4-5):727-738. doi:10.1023/A:1025048802629

33. Amaral DG, Price JL. Amygdalo-cortical projections in the monkey (Macaca fascicularis). J Comp Neurol. 1984;230(4):465-496. doi:10.1002/cne.902300402

34. Bzdok D, Laird AR, Zilles K, Fox PT, Eickhoff SB. An investigation of the structural, connectional, and functional subspecialization in the human amygdala. Hum Brain Mapp. 2013;34(12):3247-3266. doi: $10.1002 / \mathrm{hbm} .22138$

35. Roy AK, Shehzad Z, Margulies DS, et al. Functional connectivity of the human amygdala using resting state fMRI. Neuroimage. 2009;45 (2):614-626. doi:10.1016/j.neuroimage.2008.11.030

36. Amunts K, Kedo O, Kindler M, et al. Cytoarchitectonic mapping of the human amygdala, hippocampal region and entorhinal cortex: intersubject variability and probability maps. Anat Embryol. 2005;210(5-6):343-352. doi:10.1007/s00429-005-0025-5

37. Sheline YI, Gado MH, Price JL. Amygdala core nuclei volumes are decreased in recurrent major depression. Neuroreport. 1998;9 (9):2023-2028.

38. Morris JS, Buchel C, Dolan RJ. Parallel neural responses in amygdala subregions and sensory cortex during implicit fear conditioning. Neuroimage. 2001;13(6):1044-1052. doi:10.1006/nimg.2000.0721

39. Ball T, Rahm B, Eickhoff SB, et al. Response properties of human amygdala subregions: evidence based on functional MRI combined with probabilistic anatomical maps. PLoS One. 2007;2(3):e307. doi:10.1371/journal.pone.0000307

40. Whalen PJ, Kagan J, Cook RG. Human amygdala responsivity to masked fearful eye whites. Science. 2004;306(5704):2061. doi:10.1126/science.1103617

41. Bickart KC, Hollenbeck MC, Barrett LF, Dickerson BC. Intrinsic amygdala-cortical functional connectivity predicts social network size in humans. $J$ Neurosci. 2012;32(42):14729-14741. doi:10.1523/JNEUROSCI.1599-12.2012

42. Douglas N, Young A, Roebuck T, et al. Prevalence of depression in patients referred with snoring and obstructive sleep apnoea. Intern Med J. 2013;43(6):630-634. doi:10.1111/imj.12108

43. Cross RL, Kumar R, Macey PM, et al. Neural alterations and depressive symptoms in obstructive sleep apnea patients. Sleep. 2008;31 (8):1103-1109.

44. Kumar R, Macey PM, Cross RL, Woo MA, Yan-Go FL, Harper RM. Neural alterations associated with anxiety symptoms in obstructive sleep apnea syndrome. Depress Anxiety. 2009;26(5):480-491. doi:10.1002/da.v26:5

45. Kumar R, Chavez AS, Macey PM, Woo MA, Yan-Go FL, Harper RM. Altered global and regional brain mean diffusivity in patients with obstructive sleep apnea. J Neurosci Res. 2012;90 (10):2043-2052. doi:10.1002/jnr.23083

46. Yan CG, Wang XD, Zuo XN, et al. DPABI: data processing \& analysis for (resting-state) brain imaging. Neuroinformatics. 2016;14(3):339-351. doi:10.1007/s12021-016-9299-4
47. Goto M, Abe O, Aoki S, et al. Diffeomorphic Anatomical Registration Through Exponentiated Lie Algebra provides reduced effect of scanner for cortex volumetry with atlas-based method in healthy subjects. Neuroradiology. 2013;55(7):869-875. doi:10.1007/s00234-013-1193-2

48. Saad ZS, Gotts SJ, Murphy K, et al. Trouble at rest: how correlation patterns and group differences become distorted after global signal regression. Brain Connect. 2012;2(1):25-32. doi:10.1089/ brain.2012.0080

49. Maclean PD. Psychosomatic disease and the visceral brain. Psychosom Med. 1949;11(6):338-353.

50. Maclean PD. Some psychiatric implications of physiological studies on frontotemporal portion of limbic system (visceral brain). Electroencephalogr Clin Neurophysiol. 1952;4(4):407-418. doi:10.1016/0013-4694(52)90073-4

51. Auchter AM, Monfils MH. Limbic system. Int Ency Soc Behav Sci. 2015;2(14):125-130.

52. Davis M. Neurobiology of fear responses: the role of the amygdala. Neuropsychiatry Clin Neurosci. 1997;9(3):382-402. doi:10.1176/ jnp.9.3.382

53. Heimer L, Van Hoesen GW. The limbic lobe and its output channels: implications for emotional functions and adaptive behavior. Neurosci Biobehav Rev. 2006;30(2):126-147. doi:10.1016/j.neubiorev.2005.06.006

54. Price JL. Comparative aspects of amygdala connectivity. Ann N Y Acad Sci. 2003;985(1):50-58.

55. Gonzalez-Lima F, Scheich H. Classical conditioning of tone-signaled bradycardia modifies 2-deoxyglucose uptake patterns in cortex, thalamus, habenula, caudate-putamen and hippocampal formation. Brain Res. 1986;363(2):239-256.

56. Freese JL, Amaral DG. Neuroanatomy of the Primate Amygdala. The Human Amygdala. New York (NY): The Guilford Press; 2009.

57. Price JL, Drevets WC. Neurocircuitry of mood disorders. Neuropsychopharmacology. 2010;35(1):192. doi:10.1038/npp.2009.104

58. Kim MJ, Loucks RA, Palmer AL, et al. The structural and functional connectivity of the amygdala: from normal emotion to pathological anxiety. Behav Brain Res. 2011;223(2):403-410. doi:10.1016/j. bbr.2011.04.025

59. Banks SJ, Eddy KT, Angstadt M, Nathan PJ, Phan KL. Amygdala frontal connectivity during emotion regulation. Soc Cogn Affect Neurosci. 2007;2(4):303-312. doi:10.1093/scan/nsm029

60. Kohn N, Eickhoff SB, Scheller M, Laird AR, Fox PT, Habel U. Neural network of cognitive emotion regulation - an ALE metaanalysis and MACM analysis. NeuroImage. 2014;87:345-355. doi:10.1016/j.neuroimage.2013.11.001

61. Burghy CA, Stodola DE, Ruttle PL, et al. Developmental pathways to amygdala-prefrontal function and internalizing symptoms in adolescence. Nat Neurosci. 2012;15(12):1736-1741. doi:10.1038/ nn. 3257

62. Chuah LY, Chee MW. Functional neuroimaging of sleep deprived healthy volunteers and persons with sleep disorders: a brief review. Ann Acad Med Singapore. 2008;37(37):689-694.

63. Liu YT, Li HJ, Chen T, et al. Aberrant functional connectivity in patients with obstructive sleep apnea-hypopnea syndrome: a resting-state functional MRI study. Multimed Tools Appl. 2018;77 (3):4065-4079. doi:10.1007/s11042-017-4670-5

64. Squire LR, Stark CE, Clark RE. The medial temporal lobe. Annu Rev Neurosci. 2004;27(1):279-306. doi:10.1146/annurev. neuro.27.070203.144130

65. Allison T, Puce A, Mccarthy G. Social perception from visual cues: role of the STS region. Trends Cogn Sci. 2000;4(7):267-278.

66. Lim L, Radua J, Rubia K. Gray matter abnormalities in childhood maltreatment: a voxel-wise meta-analysis. Am J Psychiatry. 2014;171 (8):854-863. doi:10.1176/appi.ajp.2014.13101427

67. Nie S, Peng DC, Gong HH, Li H-J, Chen L-T, Ye C-L. Resting cerebral blood flow alteration in severe obstructive sleep apnoea: an arterial spin labelling perfusion fMRI study. Sleep Breath. 2017;21 (2):487-495. doi:10.1007/s11325-017-1474-9 
68. Chen LT, Fan XL, Li HJ, et al. Topological reorganization of the default mode network in severe male obstructive sleep apnea. Front Neurol. 2018;9:363. doi:10.3389/fneur.2018.00363

69. Heath RG, Dempesy CW, Fontana CJ, Myers WA. Cerebellar stimulation: effects on septal region, hippocampus, and amygdala of cats and rats. Biol Psychiatry. 1978;13(5):501-529.

70. Supple WF Jr, Leaton RN, Fanselow MS. Effects of cerebellar vermal lesions on species-specific fear responses, neophobia, and taste-aversion learning in rats. Physiol Behav. 1987;39(5):579-586. doi:10.1016/0031-9384(87)90156-9

71. Sacchetti B, Baldi E, Lorenzini CA, et al. Cerebellar role in fear-conditioning consolidation. Proc Natl Acad Sci U S A. 2002;99 (12):8406-8411. doi:10.1073/pnas.112660399
72. Sacchetti B, Sacco T, Strata P. Reversible inactivation of amygdala and cerebellum but not perirhinal cortex impairs reactivated fear memories. Eur J Neurosci. 2010;25(9):2875-2884. doi:10.1111/ j.1460-9568.2007.05508.x

73. Roy AK, Fudge JL, Kelly C, et al. Intrinsic functional connectivity of amygdala-based networks in adolescent generalized anxiety disorder. J Am Acad Child Adolesc Psychiatry. 2013;52(3):290-299.e2. doi:10.1016/j.jaac.2012.12.010

74. Etkin A, Prater KE, Schatzberg AF, Menon V, Greicius MD. Disrupted amygdalar subregion functional connectivity and evidence of a compensatory network in generalized anxiety disorder. Arch Gen Psychiatry. 2009;66(12):1361-1372. doi:10.1001/ archgenpsychiatry.2009.104

\section{Publish your work in this journal}

Neuropsychiatric Disease and Treatment is an international, peerreviewed journal of clinical therapeutics and pharmacology focusing on concise rapid reporting of clinical or pre-clinical studies on a range of neuropsychiatric and neurological disorders. This journal is indexed on PubMed Central, the 'PsycINFO' database and CAS, and is the official journal of The International Neuropsychiatric Association (INA). The manuscript management system is completely online and includes a very quick and fair peer-review system, which is all easy to use. Visit http://www.dovepress.com/testimonials.php to read real quotes from published authors. 